\title{
RANDOM TESTS COMBINING MATHEMATICA PACKAGE AND LATEX COMPILER
}

\author{
Emilo Gómez-Déniz ${ }^{1,2}$ Nancy Dávila-Cárdenes ${ }^{1,2}$ and $\mathrm{M}^{\mathrm{a}}$ Dolores García-Artiles ${ }^{1}$ \\ ${ }^{1}$ Department of Quantitative Methods in Economics and Management, University of Las \\ Palmas de Gran Canaria, Spain \\ ${ }^{2}$ TiDes Institute, Las Palmas de Gran Canaria, Spain
}

\begin{abstract}
This paper presents a competent and useful way to elaborate random exams by using Mathematica and LATEX. With these two tools, the authors suggest how to generate, in an easy way, different PDF documents containing different models of exams. The main idea is to provide a support to professors who have to manage groups of large number of students that should take different exams along the term, or even though not being groups of numerous students, it may be useful when different models of exams want to be provided to the students. The underlying advantage in this paper is the use of the Mathematica package for this purpose in a simple way, similarly as it has been done with alternative software. We present in this paper, some models of exams produced in the context in which the authors work.
\end{abstract}

\section{KEYWORDS}

Random test, LATEX Compiler, Mathematica software, Multiple Choice

\section{INTRODUCTION}

In Europe, the teaching-learning process has become a dynamic process mainly after the implementation of the European Higher Education Area. This fact has incorporated the continuous assessment as an essential part of the evaluation process. Nevertheless, the way in which the continuous assessment is applied or understood depends on the type of course, and in a way, on the lecturers. Some professors take into account the participation and the attendance as part of the assessment, whereas some others consider, besides the final exam, the delivery of some activities during the term. The main difficulty to implement such a system at the university is that every professor has to deal with a large number of students, at least in specific degrees like in Social Sciences (Law, Business Administration, Economics, etc.). For that reason, the continuous assessment based on different midterms tests the students should take along the course, seems to be the more extended system, at least in the university context. Based on the authors experience along years of preparing exams and exams, and being conscious the hard work involved on that, we proposed a simple process by combining two powerful tools as Mathematica software [1] and LATEX [2] for generating multiple models of exams. Some papers for generating multiple-choice tests using TeX as support may be read in [3], [4] and [5]. 


\section{METHODOLOGY}

We are going to distinguish between two areas of work: the LATEX area and the Mathematica area.

\subsection{LATEX Documents}

In order to get the target, we need the following types of files built in LATEX (we suggest to use WinEdt editor) which is a powerful and versatile all purpose text editor for Windows with a strong predisposition towards the creation of LATEX documents. This editor is available (previous fee) in the web page http://www.winedt.com/. Any other text editor may be used but the main advantage of WinEdt is that it allows us to deal different lines as just one by using wrapping (see the manual of WinEdt available in the web).

i) Firstly, although the proposed procedure would make easy to generate multiple exams, we need a bank of questions with three possible answers in which only one is correct. This bank should be organized into different topics. In our example, we have the files t_1.tex, t_2.tex, ..., t_5. These correspond to different topics of Mathematical Analysis such as limits, derivative, integration, etc. We want to point out that the order of the questions in the exam is arranged in the order in which the material is explained in class. Although the influence of the item sequence with the performance of the students on multiple-choice exams does not significantly affect students exam scores, as it has been analyzed by [6] and [7] among others. An integration question is provided as example in Figure 1.

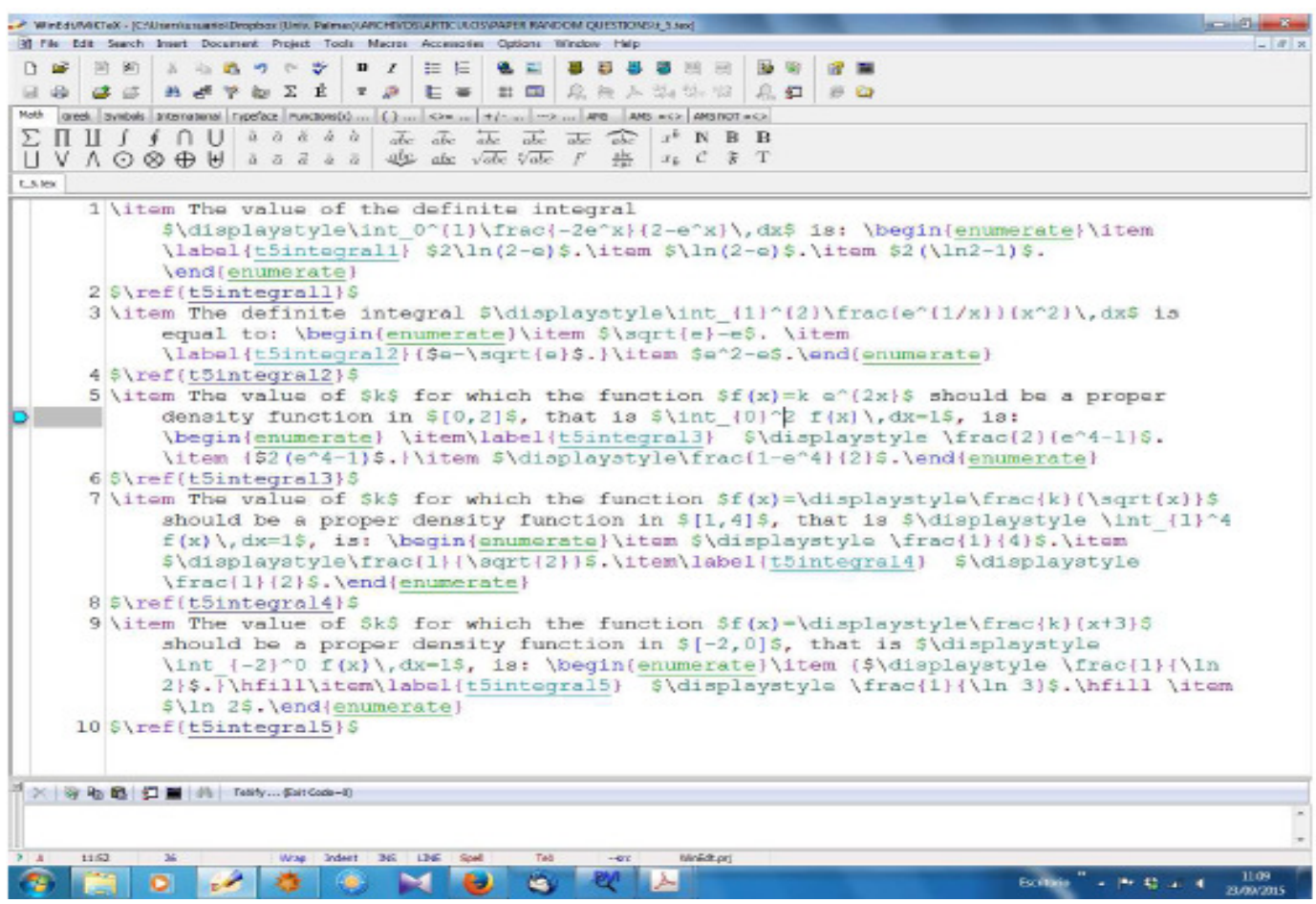

Figure 1. File t_5.tex of integration topic 
International Journal of Software Engineering \& Applications (IJSEA), Vol.7, No.4, July 2016

The advantage of using WinEdt is that it is possible to separate each question into a unique line. This is very important because next Mathematica is going to read each line as an entry in a Table. Observe that the odd lines correspond to the question (the correct answer is labelled) and the even line to the response given in a label.

ii) Secondly, EXAMEN_1.tex, EXAMEN_2.tex,..., etc. let us to generate one exam, two exams, etc. The body of these types of files is the same but different for the case of the different number of exams to generate. Here is provided an example to generate two exams.

Inewpage

lbegin \{titlepage\} Inoindent \{llarge $\mathrm{lbf}$ linput\{subject\}

\hfill $\{$ linput $\{$ date $\}\} \backslash \backslash$ linput $\{$ typeexam $\}\} \backslash\{\{$ sc linput $\{$ title $\}$

\hfill\{Type linput $\{$ type_1\}\}\}\ \I $\$

$\{$ Isc First name. \}

\hfill $\{\{\backslash \mathrm{sc}$ Surname................................... $\}\} \backslash$

lbegin $\{$ enumerate $\}$ linput $\{$ outexamen_1\}\} lend $\{$ enumerate $\}$

Ivspace $\{0.5 \mathrm{~cm}\}$

Inoindent $\{\backslash$ sf Put in the following table a $\{\backslash$ Large \$ltimes\$

in the correct answer. $\} \backslash 1 \backslash$

Large linput $\{$ tableqa $\}$

lend $\{$ titlepage $\}$

Inewpage

lbegin \{titlepage Inoindent \{llarge lbf linput\{subject\}

\hfill $\{$ linput $\{$ date $\}\} \backslash \backslash$ linput $\{$ typeexam $\}\} \backslash \backslash\{$ Isc linput $\{$ title $\}$

Ihfill\{Type linput \{type_2\}\}\}\ II II

$\{$ Isc First name.

Vhfill $\{\{\backslash$ ssc Surname...................................... $\}\} \backslash 1$

lbegin $\{$ enumerate $\}$ linput $\{$ outexamen_2\}\} lend $\{$ enumerate $\}$

Ivspace $\{0.5 \mathrm{~cm}\}$

Inoindent $\{\backslash$ sf Put in the following table a \{ $\backslash$ Large \$ltimes\$

in the correct answer. $\} \backslash \backslash \|$

Large linput \{tableqa\}

lend $\{$ titlepage $\}$

lbegin $\{$ titlepage

Ivspace* $\{2 \mathrm{~cm}\} \backslash$ noindent $\backslash$ Large

linput $\{$ degree\} $\|$ Subject: linput $\{$ subject $\} \backslash \backslash$ linput $\{$ typeexam $\}$

linput $\{$ date $\}$

Ivspace $\{1 \mathrm{~cm}\}$

Ibegin $\{$ center $\}$

\{\sc Solutions\}

lend $\{$ center $\}$

Ivspace $\{0.05 \mathrm{~cm}\}$

lbegin $\{$ table $\}[\mathrm{h}]$

lbegin $\{$ center

lbegin $\{$ tabular $\}\{\mathrm{cccccc}\}$

linput \{finaltable $\} \backslash$

lend $\{$ tabular $\}$

lend \{center\} 
lend $\{$ table $\}$

lend $\{$ titlepage $\}$

Here all the files appearing into the linput command will be generated by Mathematica and exported to the folder where we are working.

iii) Finally, the main file to be compiled in LATEX includes only a preamble (as usual in all LATEX documents) and a main body with the usual entries and an input of the file between them containing the(s) exam(s) by using inputexam, where the tex file was built automatically by Mathematica. This is given by:

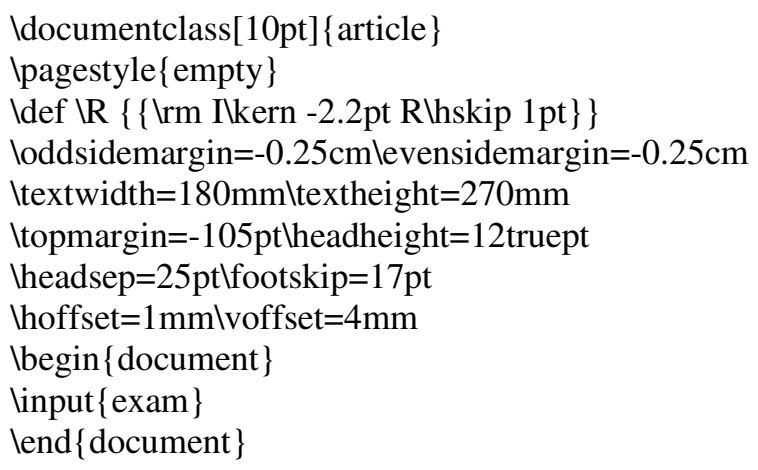

\subsection{Running Mathematica Package}

The teacher should write here the number of questions in each exam, the number of exams, etc. The example here provides five questions, two exams, etc.

\section{Mathematica Code}

numberquestions $=5 ;(*$ write the number of questions $*)$ numex $=2 ;(*$ write the number of exams to generate *) date $=$ TextForm [ "January 15_th_2015"] ;

(* write between commas the date of the exam *)

$\mathrm{s}$ u b j e c t $=$ TextForm[ "Maths"] ;

(* write between commas the subject *)

typeexam =TextForm [" First_exercise"] ;

(*write between commas the type of exam*)

degree $=$ TextForm [ "Degree_ in_B Business _ Administration "] ;

(*write between commas the degree*)

The following line builds a table where the students should put an $\mathrm{X}$ in the question they consider to be the right answer.

tableqa $=\operatorname{Grid}[$ Table $[\{\mathrm{j}, \mathrm{a}, \mathrm{b}, \mathrm{c}\},\{\mathrm{j}, 1$, numberquestions $\}]$, Divider $\mathrm{s} \rightarrow$ All $] / /$ Text ; (* build the table of responses $*$ )

We import now the type of file necessary according to the number of exams to be generated. 
Exam $=$ Import ["C: $\|$ User s $\$ Desktopl|EXAMEN "<>ToString[ numex] $<>"$. tex ", , Li s t "]

Once this is done, Mathematica export to the corresponding folder of the area of work the files built at the beginning of the Mathematica process.

Export [ "C: \I User s \Desktop \ date . tex", date , "Table"] ;

Export [ "C: \U User s IDesktoplIEXAM. tex ",Exam, "Table"] ;

Export [ "C: \\ User s \Desktop \ subject . tex", subj e c t , "Table"] ;

Export [ "C: $\|$ User s $\backslash$ Desktop $\backslash$ typeexam. tex", typeexam, "Table"] ;

Export [ "C: $\|$ User s $\mid$ Desktop $\backslash$ degree . tex ", degree , "Table"] ;

Export [ "C: $\$ User s $\|$ Desktop $\backslash$ tableqa . tex ", tabl eqa , "TeXFragment" ] ;

Now, we import the bank of questions given in files $t$ 1.tex, $t$ 2.tex, etc. and assign names to the different lines in these files. Recall that the odd lines in these files corresponds to the questions with three possible answers and the even line to the label of the correct response. These names will be used later.

a 11 q r $=$ Table [ Import [ "C: $\|$ User s $\|$ Desktop $\backslash \mathrm{t}$ " $<>$ ToString[ i ] $<>$ " tex ",

"Li s t" ], \{ i, 1, numberques tions $\}]$;

(* importing files with all topics, questions and responses $*$ )

$\mathrm{m}=$ Table $[$ Length $[$ a $11 \mathrm{q} \mathrm{r}[[\mathrm{j}]]],\{\mathrm{j}, 1$, numberques tions $\}]$;

$(*$ gives the length of topic $i *)$

a $11 \mathrm{q}=$ Table [ a $11 \mathrm{q}$ r [ [ i ] ] [ [ $2 * \mathrm{j}-1]],\{\mathrm{i}, 1$, numberques tions $\},\{\mathrm{j}, 1, \mathrm{~m}[[\mathrm{i}]] / 2\}]$; (* all questions of topic $i *)$

a $11 \mathrm{r}=$ Table $[\{$ a $11 \mathrm{qr}[[\mathrm{i}]][[2 * \mathrm{j}]]\},\{\mathrm{i}, 1$, numberques tions $\},\{\mathrm{j}, 1, \mathrm{~m}[[\mathrm{i}]] / 2\}]$

(* all responses of topic $i *)$

The main part of the Mathematica code is given next, and provides also the main LATEX documents to be compiled for giving rise to the pdf file. These main files are outexam.tex and finaltable.tex. The first file contains the exam (questions with the three possible answers) and the table with the correct responses provided to the teacher to correct the exams in an easy way.

$\operatorname{Do}[$

type $=\mathrm{j} ;\left(*\right.$ gives the version of the exam. Types $1,2, \ldots$, numex $\left.^{*}\right)$

$\mathrm{t} \mathrm{j} \mathrm{j}=$ Flatten [Table [ RandomChoice [ a $11 \mathrm{q}[$ [ i ] ] , 1 ], \{ i , 1, numberques tions $\}$ ] ] ;

(* choose random question of topic $i$ and converting

to a table withouth internal brackets *)

p t j j =Flatten [Table [ Position [ a $11 \mathrm{qr}[$ [ i ] ] t j j [ [i ] ] ] , \{ i, 1, numberques tions $\}]$ ] ;

(* position of the question chosen in the list 111 all and

converting to a table withouth internal brackets $*$ )

outexamen $=$ TableForm $[$ Table $[\mathrm{tj} \mathrm{j}[[\mathrm{i}]],\{\mathrm{i}, 1$, numberques tions $\}]]$;

(* questions chosen *)

outanswer s = TableForm[Table $[$ a $11 \mathrm{qr}[[\mathrm{i}]][[\mathrm{ptj} \mathrm{j}[[\mathrm{i}]]+1]],\{\mathrm{i}, 1$, numberques

tions \} ] ] ;

(* build the table of responses *)

Export [ "C: $\|$ User s $\|$ Desktop $\backslash$ type "<>ToString[ $\mathrm{j}]<>"$. tex ", type, "Table"] ;

Export [ "C: $\|$ User s $\backslash \backslash$ Desktop $\backslash$ outexamen "<>ToString[ $\mathrm{j}]<>$ " . tex"

, outexamen ,"Table"] ; 
Export [ "C: \| User s \|Desktop \ outanswer" $<>$ ToString[ j ] $<>$ " tex"

, outanswer s, "Table" ],

$\{\mathrm{j}, 1$, numex $\}]$;

import responses $=$ Table $[$ Import $[$ "C: $\|$ User s $\backslash$ Desktop $\|$ outanswer " $<>$

ToString[ i $]<>"$. tex ", "Li s t" ], \{ i , 1, numex $\}]$;

$\mathrm{f}$ i $\mathrm{n}$ a $1 \mathrm{t}$ a b $1 \mathrm{e}=$ TableForm[Table [ Insert [ Insert [ Insert [ Insert [

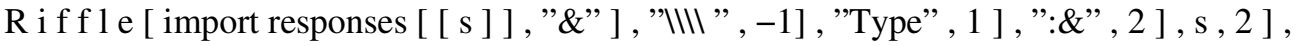

$\{\mathrm{s}, 1$, numex $\}]]$;

Export [ "C: ॥ User s \Desktop॥ f i n a 1 t a b l e . tex", f i n a 1 t a b l e, "Table "] ;

These six parts of the Mathematica code can be running together into a unique file. As the reader can see all the Mathematica commands used are simple and standard and the detailed information is provided after them as may be seen into (**). The Import command is needed to get the appropriated files, the Export to save the files generated by Mathematica and the main command to be used is Table together with Do, which uses the standard Wolfram Language iteration specification. Additionally to the mentioned files, Mathematica generates the simple files date.tex, subject.tex, typeexam.tex, degree.tex and tableqa.tex in order to print into the file EXAMEN.tex the corresponding date of the exam, the subject, the type of the exam, the degree and the table of responses where the students have to mark the responses they consider correct. Mathematica also provides the table of correct answers (responses are labelled with the corresponding labels), which is saved in the file finaltable.tex. This final table including the right answers for all the generated models might be provided also to the students, needless to say that once the examination process has finished, so they might have an idea of the score of the exam in advance. A simple chart of the process is given in Figure 2.

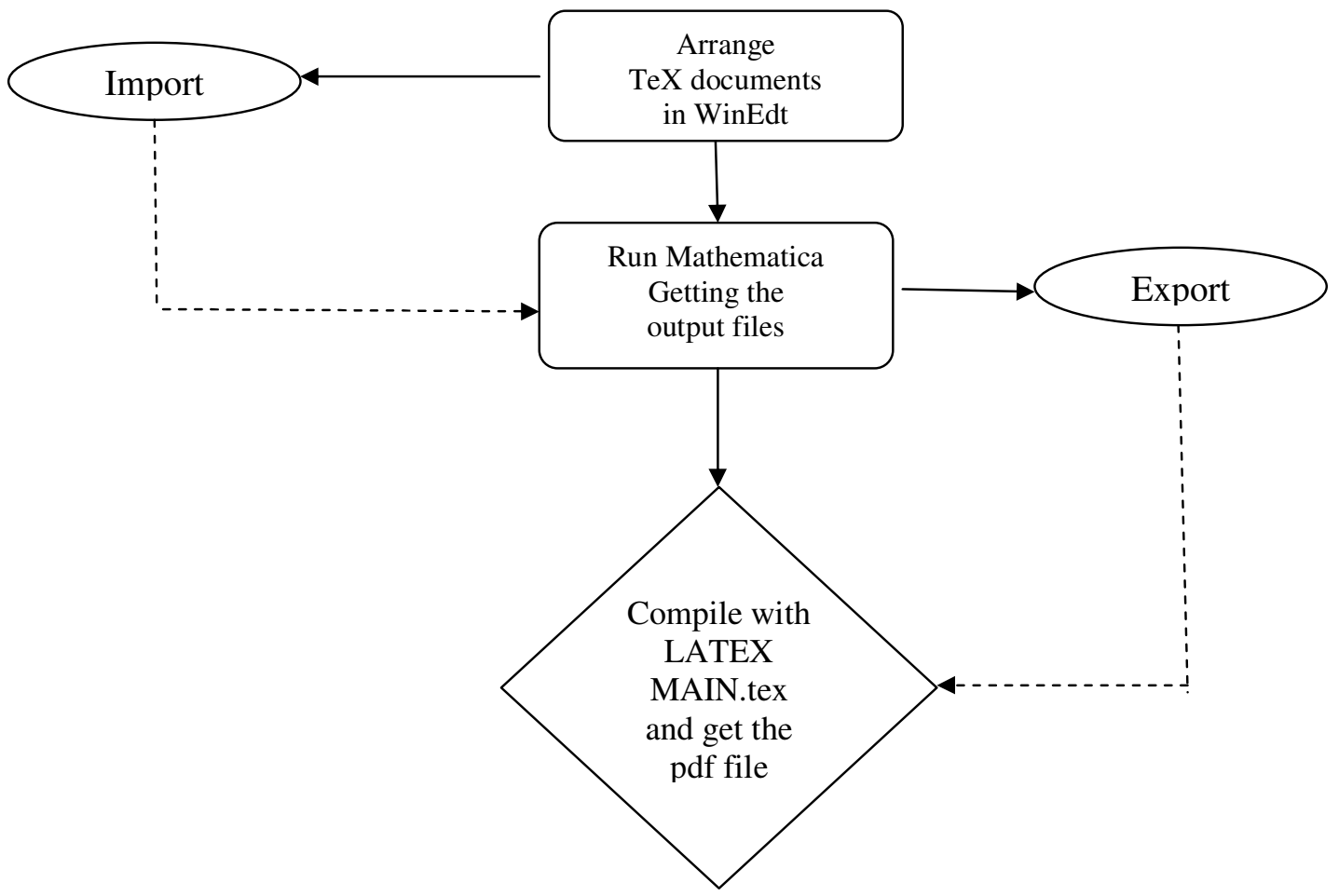

Figure 2. Chart of the process 
International Journal of Software Engineering \& Applications (IJSEA), Vol.7, No.4, July 2016

An example with two types of exams with five questions is provided and the pdf file generated is shown in Figures 3, 4 y 5.

Maths

January 15 th 2015

First exercise

TYPE 1

FIRST NAME

Surname.

1. The domain of the function $f(x)=\frac{e^{\frac{1}{x^{2}}-1}}{\sqrt{x}}$ is:
(a) $\operatorname{Dom}(f)=(0,1]$.
(b) $\operatorname{Dom}(f)=(0,1) \cup(1,+\infty)$.
(c) $\operatorname{Dom}(f)=(0,+\infty)$.

2. Given the functions $f(x)=\sqrt{x-2}, g(x)=x^{2}+1$, then the domain of the function $(f \circ g)(x)$ is:
(a) $(-\infty,-1] \cup[1,+\infty)$.
(b) $(-\infty,-1) \cup(1,+\infty)$.
(c) $\mathbb{R}$.

3. The inverse of the function $y=\frac{x+2}{x-2}$ is given by:
(a) $x=\frac{2 y+2}{1-y}$.
(b) $x=\frac{y-1}{2 y+2}$
(c) $x=\frac{2 y+2}{y-1}$

4. The function $f(x)=\frac{1+2 x}{3 x-1}$ :
(a) Is decreasing in its domain.
(b) Has a local maximum in $x=5$.
(c) Is increasing in its domain.

5. The value of the definite integral $\int_{0}^{1} \frac{-2 e^{2}}{2-e^{x}} d x$ is:
(a) $2 \ln (2-e)$.
(b) $\ln (2-e)$
(c) $2(\ln 2-1)$.

Put in the following table a $X$ in the correct answer.

\begin{tabular}{|l|l|l|l|}
\hline 1 & $a$ & $b$ & $c$ \\
\hline 2 & $a$ & $b$ & $c$ \\
\hline 3 & $a$ & $b$ & $c$ \\
\hline 4 & $a$ & $b$ & $c$ \\
\hline 5 & $a$ & $b$ & $c$ \\
\hline
\end{tabular}

Figure 3. Page 1 of the final resultant exam 
International Journal of Software Engineering \& Applications (IJSEA), Vol.7, No.4, July 2016

Maths

January 15th 2015

First exercise

TYPE 2

FirST NAME

Surname

1. The domain of the function $f(x)=\frac{\sqrt{x}}{x-1}$ is:

(a) $\operatorname{Dom}(f)=[0,1) \cup(1,+\infty)$.

(b) $\operatorname{Dom}(f)=(0,1) \cup(1,+\infty)$.

(c) $\operatorname{Dom}(f)=(0,1) \cup[1,+\infty)$.

2. Given the functions $f(x)=1-x^{2}$ y $g(x)=x+1$, then $(f \circ g)(x)$ is given by:
(a) $x(x+2)$.
(b) $2-x^{2}$
(c) $-x(x+2)$

3. The inverse of the function $y=\frac{x+2}{x-2}$ is given by:
(a) $x=\frac{2 y+2}{1-y}$
(b) $x=\frac{y-1}{2 y+2}$
(c) $x=\frac{2 y+2}{y-1}$

4. The function $f(x)=\left(x^{2}-3\right) e^{2}$ is:
(a) Decreasing in en $(-3,1)$.
(b) Decreasing in $(-\infty,-3) \cup(1,+\infty)$.
(c) Increasing in $(-3,+\infty)$.

5. The value of $k$ for which the function $f(x)=\frac{k}{\sqrt{x}}$ should be a proper density function in $[1,4]$, that is $\int_{1}^{4} f(x) d x=$ 1 , is:
(a) $\frac{1}{4}$.
(b) $\frac{1}{\sqrt{2}}$.
(c) $\frac{1}{2}$.

Put in the following table $a \mathrm{X}$ in the correct answer.

\begin{tabular}{|l|l|l|l|}
\hline 1 & $a$ & $b$ & $c$ \\
\hline 2 & $a$ & $b$ & $c$ \\
\hline 3 & $a$ & $b$ & $c$ \\
\hline 4 & $a$ & $b$ & $c$ \\
\hline 5 & $a$ & $b$ & $c$ \\
\hline
\end{tabular}

Figure 4. Page 2 of the generated exam in the process 
Degree in Business Administration

Subject: Maths

First exercise January 15 th 2015

\section{Solutions}

Type 1: $1 c \quad 2 a \quad 3 c \quad 4 a \quad 5 a$

Type 2: $1 a \quad 2 c \quad 3 c \quad 4 a \quad 5 c$

Figure 4. Page 3 of the generated solution table in the process

\section{Conclusions}

In this work, we provide an effective way to elaborate several models of multiple-choice tests (essays type are also possible) by using Mathematica and LATEX. The combined use of these two tools allow us to generate in an easy way, an assorted set of different PDF documents containing different versions of the same exam, in the case of multiple-choice tests, a table containing the solutions is provided for making easy the process of correction. The questions on the generated exams include the topics in the same order theyare presented in the contents of the course because in this way it has been decided at the moment of designing the output of the program but this order could be scrambled. Depending on the bank of questions for each topic, the probability of repeating questions is lower. With regard to the answers, as the right questions are labeled the order in which they are shown could be scrambled with the automatic change in the complete solution table provided to the students in order they could have an idea of their score. Finally, the purpose of the authors with this paper is to provide for Mathematica users a simple tool that help to save time for preparing a collection of several models of exams, as the users of alternative software have done

\section{REFERENCES}

[1] Wolfram, S. (1996) The Mathematica Book, Third Edition. Cambridge University Press.

[2] Mora, F.W. and Borbón A. A. (2013). Edición de Textos Científicos LATEX2014. Revista digital Matemática. Educación e Internet.

[3] Olimpia, C and Mircea, D.(2012). "Radomly Generated Multiple-Choice Tests Using (La)Tex and Pseudorandoms Number Generators". Bulletin UASVM Horticulture, 69(2) pp537-538.

[4] Sojka, P. (2003) "Rapid Evaluation using Multiple Choice Tests and. TeX". ITiCSE'03. Thessaloniki, Greece.

[5] Saludes, J.(1996) "Fast and secure Multiple-Options Tests", TUGboat, Vol 17, No. 3 pp 310-319.

[6] Carlson, J.L. and Ostrosky, L. (1992). "Item Sequence and Student Performance on Multiple-Choice Exams: Further Evidence”. The Journal of Economic Education, Vol. 23, No. 3, pp232-235.

[7] Caudill, S.B. and Gropper D.M. (1991). "Test Structure, Human Capital, and Student Performance on Economics Exams". The Journal of Economic Education, Vol. 22, No.4, pp303-306. 
International Journal of Software Engineering \& Applications (IJSEA), Vol.7, No.4, July 2016

\section{Authors}

Emilio Gómez Déniz. Ph. D. in Economics and Business Management. Chair professor with at the University of Las Palmas de Gran Canaria (Spain) in Mathematic for Business. Research focused on Bayesian and Actuarial Statistics and in the areas of Teaching Methodologies and Educational Innovation. Member of the Institute of Tourism and Sustainable Economic Development (TiDES).

Nancy Dávila Cárdenes. Ph. D. in Economics and Business Management. Full professor at the University of Las Palmas de Gran Canaria (Spain) in Mathematic for Business. Research focused on Dynamic Optimization and more recently in the areas of Teaching Methodologies and Educational Innovation. Manager of the Institute of Turism and Sustainable Economic Development (TIDES).

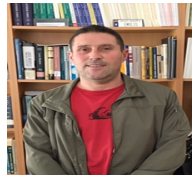

$\mathrm{M}^{\mathrm{a}}$ Dolores García Artiles. Ph. D. in Economics and Business Management. Full professor at the University of Las Palmas de Gran Canaria (Spain) in Mathematic for Business. Research focused on Non-Linear Dynamics and Finance, Computational Quantitative Finance and Models of Educational Innovation and Teaching Methodologies.
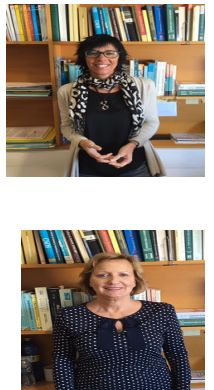